\title{
Robot-assisted laminectomy in spinal surgery: a systematic review
}

\author{
Zhuofu $\mathrm{Li}^{1,2,3} \wedge$, Guoxin $\mathrm{Yu}^{4}$, Shuai Jiang ${ }^{1,2,3}$, Lei Hu${ }^{4}$, Weishi $\mathrm{Li}^{1,2,3} \wedge$ \\ ${ }^{1}$ Department of Orthopaedics, Peking University Third Hospital, Haidian, Beijing, China; ${ }^{2}$ Beijing Key Laboratory of Spinal Disease Research, \\ Haidan, Beijing, China; ${ }^{3}$ Engineering Research Center of Bone and Joint Precision Medicine, Ministry of Education, Beijing, China; ${ }^{4}$ Robotics \\ Institute, Beihang University, Beijing, China \\ Contributions: (I) Conception and design: All authors; (II) Administrative support: W Li, L Hu; (III) Provision of study materials or patients: Z Li, G \\ Yu, S Jiang; (IV) Collection and assembly of data: Z Li, G Yu; (V) Data analysis and interpretation: Z Li, G Yu; (VI) Manuscript writing: All authors; \\ (VII) Final approval of manuscript: All authors. \\ Correspondence to: Weishi Li. Department of Orthopaedics, Peking University Third Hospital, 49 North Garden Road, Haidian District, Beijing \\ 100191, China. Email: puh3liweishi@163.com.
}

\begin{abstract}
This study aimed to summarize the current progress in the field of robot-assisted laminectomy in spinal surgery. A systematic search of the Institute of Electrical and Electronics Engineers (IEEE) Xplore, PubMed, Embase, Web of science, The Cochrane Library, Wanfang Data, China National Knowledge Infrastructure (CNKI), and Chinese Biomedicine Literature Database (CBM-SinoMed) was performed for papers related to robotic-assisted laminectomy. A total of 27 articles were selected for inclusion in our study. Among the articles, 10 robotic system, 2 bone cutting strategies, 6 state recognition strategies were founded. The most commonly adopted type of robot system was the Nathoo A type (6/10). Bone cutting strategies were mainly formulated based on force information and medical image information, and state recognition was based on a variety of factors, including force, sound, vibration, medical images, current or a combination of parameters. Early research on robot-assisted laminectomy did not reflect good continuity, and the studies mainly focused on the type of robotic system. In recent years, more researchers have chosen the Nathoo A as the robot system type, and the focus of research has gradually shifted to laminectomy path planning and safety control strategies, such as state recognition. Although these studies have been able to perform laminectomy without penetrating the inner cortex of the lamina, most experiments have been performed in vitro, and clinical application is still untested.
\end{abstract}

Keywords: Robot-assisted surgery; laminectomy; state recognition; bone cutting strategy

Submitted Jul 12, 2020. Accepted for publication Feb 04, 2021.

doi: $10.21037 /$ atm-20-5270

View this article at: http://dx.doi.org/10.21037/atm-20-5270

\section{Introduction}

More than 4.83 million people undergo spinal surgery each year (1). With an aging population, it is expected that the number of patients suffering from degenerative spinal diseases will increase, and this will, to an extent, increase the amount of spinal surgery in turn. Performing spinal surgeries with high efficiency and quality is a challenge for spine surgeons in the new era. One solution has been offered in the form of robot-assisted spine surgery. In the past few decades, surgical robots aimed at reducing human error and improving the safety and efficiency of surgery have developed rapidly in fields like general surgery and urology $(2,3)$. However, the development of surgical robots in the spine field has been relatively slow. So far, the United

^ ORCID: Zhuofu Li 0000-0001-5839-2447; Weishi Li 0000-0001-9512-5436. 
States Food and Drug Administration (FDA) has approved a total of 7 robots from 4 companies (4), and the China Food and Drug Administration (CFDA) has approved 1 robot for spinal surgery. Those robots mainly address the accuracy and safety problems of pedicle screw placement through navigation and positioning. However, in the field of robotassisted laminectomy, there are no commercially available robots, and most studies are in the experimental stage. Laminectomy is a common operation in spinal surgery and even neurosurgery. It has a wide range of indications in diseases such as disc herniation, spinal stenosis, and spinal tumor. Research in this field has favorable prospects in clinical application. However, there is a lack of a review in the field of robot-assisted laminectomy. Therefore, we performed a systematic review of the current research progress in this field. We present the following article in accordance with the PRISMA reporting checklist (available at http://dx.doi.org/10.21037/atm-20-5270).

\section{Methods}

We performed a systematic literature search for all relevant articles in English or Chinese language from the Institute of Electrical and Electronics Engineers (IEEE) Xplore digital library, PubMed, Embase, Web of Science, The Cochrane Library, Wanfang Data, China National Knowledge Infrastructure (CNKI), and the Chinese Biomedicine Literature Database (CBM-SinoMed), limiting the publication date from inception to March, 2020. We used boolean operators to link Medical Subject Heading (MESH) terms, including "decompression", "laminectomy", "laminotomy", and "robot". Specific strategies were made to fit features of the different bibliographic databases (see Table S1). In addition, reference lists were manually examined for additional studies not retrieved from database searches. The search was performed at the same time by 2 researchers, and disagreements were resolved through discussion with a third researcher. Only articles which studied "robotic-assisted laminectomy" in spine surgery were included.

\section{Results}

After the literature and application of the inclusion criteria, 27 articles were selected for study inclusion (Figure 1). All articles included were published between 2001 and 2020 . There were 3 articles from 2000 to 2004, 2 from 2005 to 2009, 5 from 2010 to 2014, and 17 articles from 2015 to
2020. The articles mainly originated from 4 countries: China ( $\mathrm{n}=20$ articles), France ( $\mathrm{n}=5)$, Germany $(\mathrm{n}=1)$, and the United States ( $\mathrm{n}=1$ article). All articles published in the past 5 years were from China (Figure 2). The data regarding the robot system, bone cutting strategy, and state recognition strategies are summarized below.

\section{Robotic system classification}

In 2005, Nathoo et al. (5) proposed a 3-type classification system for surgical robots. (I) The supervisory controlled robotic system involves the surgeon completing a surgical plan before the operation, with the robot automatically executing the plan under the supervision of the surgeon during the operation. (II) The robotic telesurgical system, is considered to operate under a "master-slave" condition, an example of which is the da Vinci surgical system. In this system, there is a master side and a slave side, and, during the operation, the slave-side mechanical arm imitates the doctor's operation on the master-side. Finally, (III) the shared control system involves the surgeon and the robot controlling the surgical instrumentation at the same time. For the purposes of this study, the robotic systems were summarized according to the Nathoo classification (Table 1).

\section{Nathoo type A: supervisory controlled robotic system}

In 2010, Wang et al. (6) designed a compact spinal milling robot (SMR). The robot consists of a 2 degrees-of-freedom (DOF) feeding unit and a milling unit which contains a force sensor. The former is used to complete the translational feeding motion, while the latter can perform the rotational cutting process and force monitoring function. The milling terminal is a spherical milling cutter (Figure 3). In 2015, Dai et al. (7) developed a robotic system consisting of a 3-DOF robot, a non-contact laser displacement sensor, a water cooling tube, and a spherical milling cutter. The laser displacement sensor is used to detect the vibration of the spinous process during the laminectomy to achieve state recognition. They also applied this robotic system several times to study different methods of state recognition during laminectomy (16-18). In 2010, Jin et al. (19) introduced a pedicle screw surgical robot system, which can be used to drill and place screws in pedicles automatically; this then gradually developed into the robotic spinal surgical system (RSSS) (20,21). In 2013, Deng et al. (8) modified the RSSS and applied it to the field of laminectomy. The modified system includes a 7-DOF manipulator in which the 


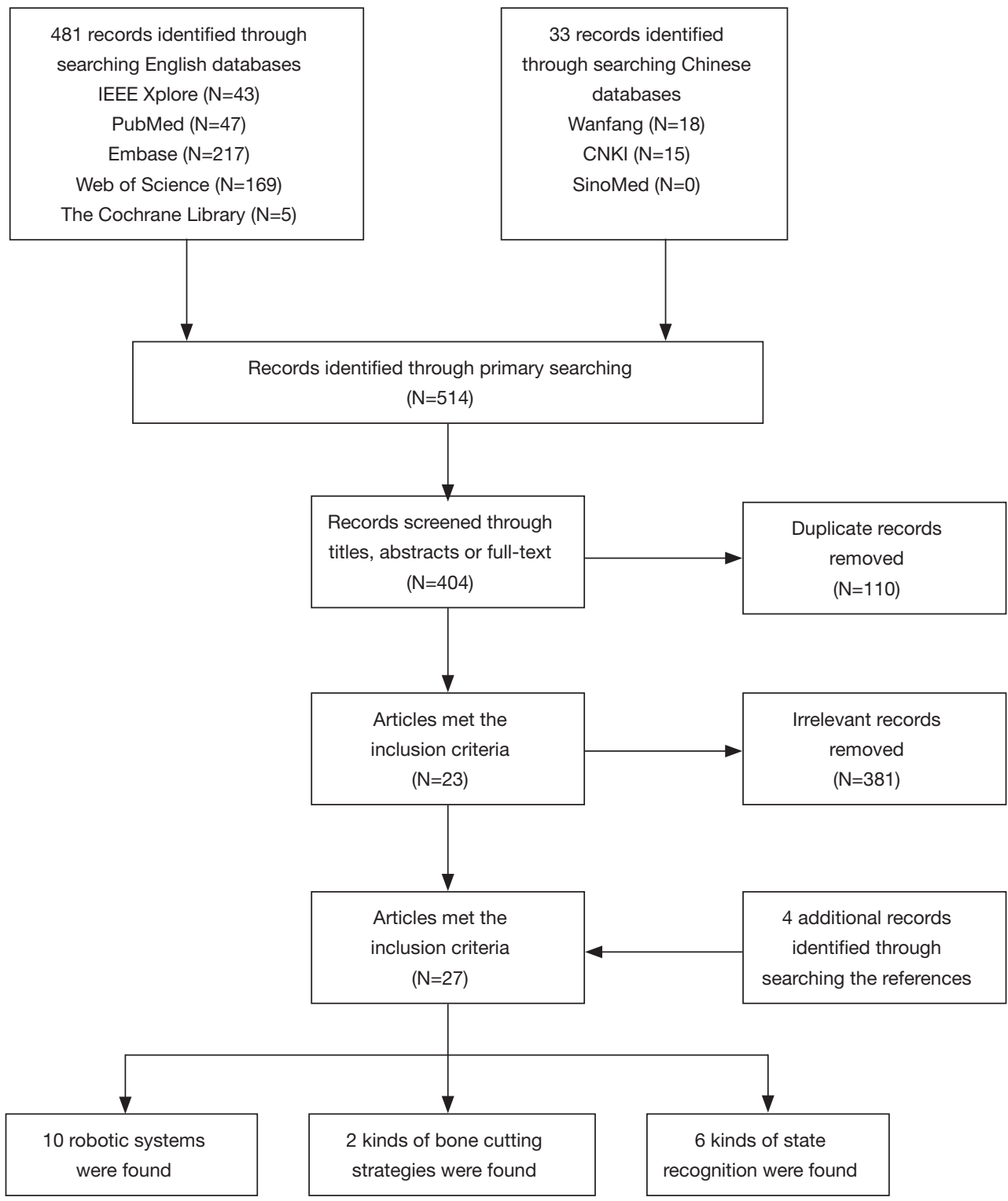

Figure 1 Flowchart of selection of robot-assisted laminectomy studies.

terminal has a spherical milling cutter with a force sensor. During the operation, the lateral interaction force of the milling cutter is collected by the force sensor and analyzed to ascertain the milling state. In 2016, Deng et al. (11) also used a 3-axis robot system to perform laminectomy, which combined with a force/torque sensor, with the milling terminal being a spherical milling cutter. In 2017, Wang et al. $(22,23)$ introduced the design scheme for a laminectomy robot composed of a 5-DOF robotic arm and a feeding unit. In 2018, Sun et al. (24) introduced the surgical robotic auxiliary system (SRAS). The system consists of a universal robot UR5 mechanical arm (6DOF), a three-dimensional (3D) image interaction using Visualization ToolKit/Insight ToolKit (VTK/ITK) libraries, and probe tracking through serial communication.

In 2016, Wang et al. and Fan et al. $(9,25)$ introduced a vertebral milling surgical robot system, with explanations of the preoperative to intraoperative procedures. This system comprises an image navigation system and a robot motion control system. The former includes an infrared optical locator and navigation software for surgical path planning and intraoperative real-time tracking and positioning. 
The latter consists of a robot control interface and a surgical robot (a 4-DOF robotic arm, a 6D force/torque sensor, and a spherical grinding drill) for positioning and grinding operations. In this system, computed tomography (CT) images are acquired before surgery to reconstruct a $3 \mathrm{D}$ model of the spine. After registration, the surgeon formulates the operation plan according to the $3 \mathrm{D}$ model, and finally the robot automatically performs the laminar milling operation according to the plan. The author conducted a total of 12 experiments on pig, sheep, and bovine spines. The results showed that the remaining thickness of the lamina was 1-2 mm, and the success rate of the experiment was $100 \%$.

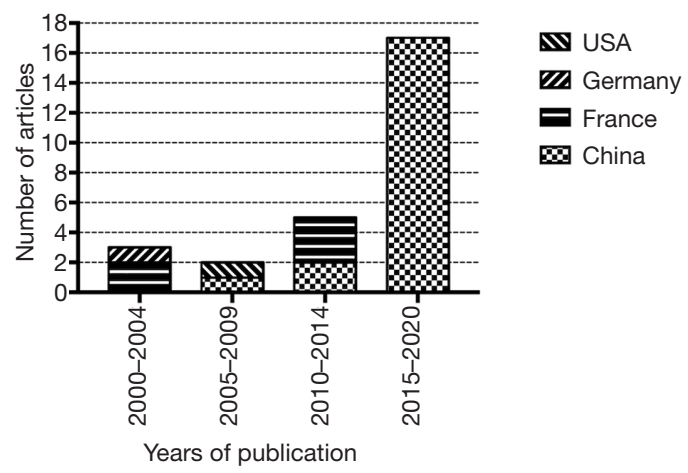

Figure 2 A breakdown of articles published per 5 years from 2001 to 2020 .

\section{Nathoo type B: robotic telesurgical system}

In 2009, Ponnusamy et al. (12) reported use of the da Vinci surgical system for posterior spinal surgery including laminectomy. The da Vinci surgical system has been widely used in urology and gynecology operations (26). However, due to the lack of bone resection equipment, its application in spinal surgery is limited (27). Ponnusamy et al., installed prototype burr and rongeur instruments to the da Vinci robot component, and performed posterior spinal surgery on pigs. In this operation, the da Vinci robot is placed vertically in the operation area, and the robot is operated remotely for laminotomy, laminectomy, and dural suture. There was no unexpected instrument movement during the bone resection, and the laminectomy was successful. In 2016, Lei et al. (13) built a master-slave spine surgery robot system. The master end of the system is composed of omega.6 force-feedback equipment (Force Dimension, Nyon, Switzerland), master console, host, and display. The slave end includes the spine surgery robot arm, camera, milling drill, and 6D force sensor. During the operation, the surgeon operates the movement of the master hand, and then the system maps the operation of the surgeon to the slave arm, and controls the slave arm to perform the operation. At the same time, the system collects the milling force information from the force sensor to provide force feedback to the master hand. The experimental results showed that the motion error of the master and slave hands varied within $\pm 1 \mathrm{~mm}$.

Table 1 Summary of robotic systems for laminectomy

\begin{tabular}{llllll}
\hline Name (or reference) & Type* & DOF & State recognition & Cutting strategy & Reference \\
\hline SMR & A & 2 & Force & Layer by layer & Layer by layer \\
Dai 2015 & A & 3 & Vibration & Layer by layer & $(7)$ \\
RSSS & A & 7 & Force & Layer by layer \\
Wang 2016 & A & 4 & Force & Medical Image \\
SRAS & A & 6 & Location & Fuzzy force control \\
Deng 2016 & A & 3 & Force & Remote operation \\
da Vinci & B & 6 & N/A & Remote operation \\
Lei 2016 & B & 6 & Force & Collaboration \\
Hein 2001 & C & N/A & N/A & Collaboration \\
Surgicobot & C & N/A & N/A & $(12)$ \\
\hline
\end{tabular}

*Nathoo type. DOF, degree of freedom; SMR, spinal milling robot; RSSS, robotic spinal surgical system; SRAS, surgical robotic auxiliary system. 


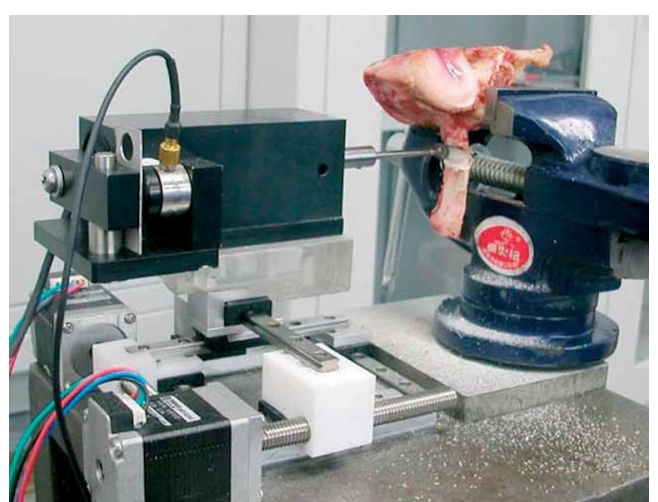

Figure 3 Spinal milling robot designed by Wang et al. (6) consisting of a 2-DOF feeding unit and a milling unit. Permission to reproduce this figure is obtained from copyright holder. DOF, degree of freedom.

\section{Nathoo type C: shared control system}

In 2001, Hein et al. (14) introduced a surgical robot that can restrict the movement of the operating terminal during operation. This robot calibrates the safety boundary in advance, and the surgeon can only move the terminal within a safe range, thereby ensuring the safe control of laminectomy. Similarly, in 2004, the The French Atomic Energy Commission designed a collaborative robotic system, Surgicobot $(15,28-30)$. This system is installed on a bridge spanning the operating table, and consists of a 6-DOF tandem manipulator, a positioning camera, cutting tools, probes, and a graphical interface for human-machine interaction (31). Before surgery, the $3 \mathrm{D}$ model of the vertebral body is extracted from the CT image to define the "danger area". Then, the surgeon's operation is constrained by the force generated by the robotic arm, and will not be able to penetrate the danger area of the patient's in surgery.

\section{Bone cutting strategy}

The bone cutting strategy is formulated to select the most efficient way to perform laminectomy. For Nathoo B and Nathoo C robots, the intraoperative bone cutting strategy is the same as that in traditional surgery. For Nathoo A robots, it is necessary to develop a bone cutting strategy, as the cutting process is controlled by the robot.

\section{Medical image-based strategies}

In 2018, Sun et al. (24) formulated a cutting strategy based on the 3D reconstruction of medical images and the iterative closest point (ICP) space registration algorithm. In this strategy, the doctor plans the cutting path on the interactive interface. The robot system generates the upper and lower surface points of the area to be cut, and then uses the binary space partitioning-tree (BSP tree) algorithm to generate the middle part point set. Finally, the robot system generates the cutting path which is converted into the robot space. In order to generate the speed of the corresponding point of the path, the mapping relationship between the gray value of the medical image and the virtual force value is found through calculation based on the medical image. The virtual force of the next position is calculated before the robot moves to adjust the cutting parameters and improve the cutting effectiveness. An experiment on the scapula of pigs showed that the remaining thickness after laminectomy was about $1 \mathrm{~mm}$, with the inside of the lamina being left unpenetrated.

\section{Force-based strategies}

Zhang et al. (32) introduced a fuzzy logic-based control method, which uses the force and the thickness of the lamina as input to output the cutting depth and speed. The fuzzy control rule is based on the doctor's experience and experimental results. Matrix laboratory (MATLAB) was used for simulation experiments and verified the feasibility of the control system. Qi et al. (33) and Ma (34) used a multilevel fuzzy controller (MLFC) to adjust the transverse feed speed in real time based on force feedback. In the experiment, the feed rate was selected to be $1 \mathrm{~mm} / \mathrm{s}$ for conventional grinding, and was adjusted in real time for MLFC grinding. The time consumption of MLFC grinding was reduced by more than $45 \%$ compared with conventional grinding. The coefficient of variation of longitudinal force was reduced by $80 \%$ on average, which indicated that MLFC grinding has higher cutting efficiency and is more stable. Fan et al. (35) input the filtered force signal into the fuzzy logic controller (FLC) to output the cutting depth, and adjust the cutting depth in real time during the milling process. In an experiment that tested how many layers there were before it milling to the inner cortex, the normal milling had 10 layers, while the FLC had 7, indicating the latter had higher efficiency. Deng et al. (11) used damping control (DC) to adjust the feed speed according to the lateral force feedback, stiffness control (SC) to adjust the cutting depth according to the longitudinal force, and a fuzzy combiner (FC) to adjust the cutting parameters in real time. They compared 
Table 2 Summary of state recognition strategies

\begin{tabular}{|c|c|c|c|}
\hline Signal & Sensor & Model & Reference \\
\hline \multirow{3}{*}{ Force } & \multirow{3}{*}{ Force sensor } & Normalized mean feature & (35) \\
\hline & & Hilbert-Huang transform, support vector machine & (8) \\
\hline & & Energy consumption & (11) \\
\hline \multirow[t]{2}{*}{ Sound } & \multirow[t]{2}{*}{ Sound sensor } & Wavelet packet transform, self-organizing feature mapping & $(17)$ \\
\hline & & Discrete wavelet transform & (37) \\
\hline Vibration & Laser displacement sensor & Wavelet packet transform, adaptive linear neuron & (7) \\
\hline Location & $\mathrm{CT}$, navigation system & U-Net, gray redistribution & $(10)$ \\
\hline Multi-information & $\begin{array}{l}\text { Acceleration sensor and laser } \\
\text { displacement sensor }\end{array}$ & Lifting wavelet package transform, support vector machine & $(16)$ \\
\hline
\end{tabular}

$\mathrm{CT}$, computed tomography.

3 milling methods: uncontrolled, force-controlled, and fuzzy force-controlled milling. The experiment showed that the longitudinal force of fuzzy force-controlled milling changed by about $26 \%$ and the other 2 methods exceeded $50 \%$. The time for fuzzy force control was about 40 seconds, while that of free control and force control was 60 seconds and 45 seconds, respectively, indicating that the fuzzy force control was more efficient.

\section{State recognition strategy}

State recognition means that the robot recognizes the operation condition of the bone cutting terminal during operation. It is critical to be able to identify the inner cortical bone, so as to stop grinding when coming into contact with this bone. The currently used state recognition methods are summarized in Table 2.

\section{Force-based state recognition}

In 2010, Wang et al. (6) used the sudden decrease of the lateral force when milling to the edge of the lamina as an indicator for stopping grinding, and differentiated the cortical from cancellous bone by calculating the crosscorrelation coefficient (CCC). The tests showed that the remaining bone thickness was $0.9-1.2 \mathrm{~mm}$, with none of test procedures having broken through the inner cortical layer. In 2016, Fan et al. (35) used longitudinal contact force to assess the milling state. They used a normalized mean feature (NMF) algorithm on longitudinal forces and found that the normalized force in cancellous bone was $0.4-0.5$, while that of milling in cortical bone was higher than 0.9 . The difference was obvious, and the milling state could be identified. The results of this experiment showed that the remaining lamina thickness was between $1.0 \mathrm{~mm}$ and $1.9 \mathrm{~mm}$. In 2013, Deng et al. (8) used the Hilbert-Huang transform (HHT) to extract force features by collecting lateral forces during the grinding process, and used support vector machine (SVM) to establish classifications for identifying different milling stages. Experiments showed that the correct recognition rates of the outer cortical bone, cancellous bone, and inner cortical bone were $86.7 \%$ (13/15), 86.7\% (13/15), and 100\% (15/15), respectively. In 2016, Deng et al. (11) calculated the energy consumption (EC) of the milling process by obtaining the contact force, feed speed, and cutting depth during the cutting process. Compared with cancellous bone, cortical bone consumed more energy, so the difference between cancellous and cortical bone can be identified according to the EC value. Using this method, the milling tool can stop when 
touching the inner cortical bone with a residual thickness of 1.9-2.2 mm. In 2020, Jiang et al. (36) built a milling dynamics model based on the geometry theory of the ball-end cutter, and used the particle swarm optimization algorithm (PSO) to calculate the correlation coefficient of the milling dynamics model to calibrate the model. The experimental results show that the prediction accuracy was $\pm 0.1 \mathrm{~mm}$ and within $0.8-1.2 \mathrm{~mm}$, while the accuracy was $\pm 0.2 \mathrm{~mm}$ and within $1.2-1.6 \mathrm{~mm}$.

\section{Sound-based state recognition}

In 2015, Dai et al. (37) obtained the sound signal during milling process through a microphone, analyzed the sound pressure signal using discrete wavelet transform (DWT) to extract the harmonic component, and calculated the product of wavelet energy to identify different milling states. In tests, the doctor performed laminectomy by hand, and calculated the product of wavelet energy of the 5 operating states: idling, start, 2 seconds before breaking through, 1 second before breaking through, and broken through. The result showed that the difference in the wavelet energy produced was obvious. In 2017, wavelet packet transform (WPT) was performed on the sound pressure information collected during the milling process, and a series of sub-band signals was decomposed (17). Then, the average amplitude of the sub-band wavelet coefficients was calculated, and the result was input to the self-organizing feature mapping network (SOFM) for classification and recognition. The SOFM output 4 operation results: nothing, cortical bone, cancellous bone, and annulus fibrosus. The experimental results show that the successful recognition of the 4 states was not less than $85 \%$.

\section{Vibration-based state recognition}

Dai et al. (7) used vibration harmonic amplitudes to identify different milling states. They acquired vibration displacement signals through laser sensors and performed median filtering, then performed WPT, and finally used adaptive linear neuron (ADALINE) to obtain the harmonic amplitude. The different milling states were identified by the harmonic amplitude. The experiment was consistent with the dynamic analysis results, and harmonic amplitude was found to be able to monitor the key state before breakthrough. The remaining thickness of the lamina was maintained at about $1 \mathrm{~mm}$.

\section{Medical image-based state recognition}

Sun et al. (10) registered the medical image space and the navigation space, and identified different operating states based on the gray value of the medical image during the operation. In order to improve the recognition of the lamina edge, the medical image was preprocessed. First, the U-Net network was used to perform binary segmentation on the medical image, and then the grayscale redistribution method was used to enhance the lamina edge information, which allowed the robot to identify the inner cortex during fenestration. Simulation experiments were carried out. The results showed that the lamina was not broken through under different speeds and different dynamic receiving field conditions.

\section{Current-based state recognition}

Zhu et al. (38) collected the current signal during the milling process, normalized it by wavelet transform, and obtained the converted current signal. The milling state was determined according to the difference between this current value and the threshold. The experiment showed that the remaining thickness of the lamina was 0.98-1.84 mm.

\section{Multi-information-based state recognition}

Researchers have also tried to use multi-information for state recognition. Dai et al. (18) obtained 2 signals through sound sensors and acceleration sensors, used a band-pass filter to extract the harmonics of the 2 signals, calculated the correlation coefficients between the 2 harmonics, and finally input them into the artificial neural network (ANN). The model output 4 cutting states: cancellous bone, cortical bone, muscle, and idling. The recognition accuracy of the experimental model was more than 90\%. In 2016, Dai et al. (16) used acceleration sensors and laser displacement sensors to obtain vibration signals of tools and tissues. First, they used a median filter for the initial signals, then input the results into the lifting wavelet packet transform (LWPT) model, and finally input the calculated wavelet factor into the SVM classifier. As a result, the classifier output the state recognition results, which were the lamina, adjacent bone structure, muscle, and spinal cord. The success rate of the identification of adjacent bone structure and muscle was about $90 \%$. 


\section{Discussion}

The robots in the field of spinal surgery can be divided into pedicle screw fixation robots and laminectomy robots. Many supporting studies and successfully commercialized products exist for pedicle screw fixation. The FDA has approved a total of 7 robot type from 4 companies, while the CFDA has only approved 1 robot (TiRrobot system, TINAVI Medical Technologies Co., Ltd, Beijing, China). The Mazor SpineAssist ${ }^{\circledR}$ (Mazor Robotics Ltd.; Caesarea, Israel) robotic system, the first of its kind to be approved by the FDA, went on the market in 2004, while the first generation TiRobot system was licensed by the CFDA in 2010. Despite its relatively late start, the TiRobot is in a leading position in the market to some extent, due to it having obtained indications for the whole spine and trauma (39), and its positioning accuracy has reached the sub-millimeter level. These commercial robots are not capable of autonomous operation. Another robot, SPINEBOT, proposed by Hanyang University in South Korea in 2005 (40), has auto-drilling functionality, and simulation test results have shown an error range within $2 \mathrm{~mm}$. On this basis, in 2009, a team from the Pohang University of Science and Technology in South Korea designed the cooperative robotic assistant (CoRA) robot (41); this system had a rigid body to better withstand the reaction force in the process of drilling and nailing, but also had the disadvantage of being too large in size. In 2011, a Chinese team proposed the robot spinal surgical system (RSSS) (20), which was later iterated to the RSSSII. The serial structure was adopted to effectively reduce the volume, and the experiment supported its accuracy: the average positioning error was $1 \mathrm{~mm}$ and the angle error was 1.1 degrees. Evidently, compared with other countries, China was late to engage in this field, with fewer commercial products and fewer practical clinical applications. In addition to pedicle screw fixation, laminectomy is another critical step for spine surgery. Robot-assisted laminectomy has gradually attracted the attention of scholars in recent years, especially those from China (of the 27 studies included in this paper, 20 are by Chinese researchers). The focus of Chinese researchers is different from that of other countries, where the early research still focuses on 2 types of robots: remote operation and collaborative robots. The content of Chinese research is focused on methods to perform autonomous robotic laminectomy, with the degree of automation of the robot being obviously higher. Currently, there is no successful commercial product of a laminectomy-performing surgical robot, and this vacancy represents an opportunity for China gain an edge in the high-end medical equipment market.

In the field of robot-assisted laminectomy, 3 kinds of robotic systems exist. Among them, the Nathoo A type is the most commonly used, which also in line with the public's expectations for surgical robots; that is, a robot capable of automatically performing surgical operations. Robots of this type generally share same characteristics in hardware construction. The operation port contains a multi-DOF mechanical arm, which holds the bone cutting tool, and integrates different sensors according to need. The control port regulates operation of the robot arm by analyzing the signals collected by the sensor. The robot's safety strategy is to use various signals to identify the inner cortical bone during milling procedures, so that it can stop before penetrating the inner cortical bone, avoiding damage to the vital tissues. However, related research has only been performed using in vitro bone specimens or bone models. Future studies need to further examine the interfering factors of the in vivo environment, such as the patient's respiration, influence of surgical field bleeding, possible elastic deformation of the lamina (42), or even displacement of the vertebral body due to the excessive force between the bone cutting tool and the lamina.

The Nathoo B robot requires the full participation of the surgeon during the operation. The robot itself does not determine the progress of the operation. The advantage is that the slave port can be designed to be maximally conducive to the purpose of the operation, can perform surgery in a cramped surgical space (such as in minimally invasive surgery), and offer a comfortable operating environment for the surgeon. However, as far as the current research is concerned, these advantages have not been well demonstrated during the operation of laminectomy. This may be because current research is still limited to open surgery or in vitro experiments, which cannot showcase the advantages of minimally invasive surgery. Additionally, due to the large size of the robot's slave port, its placement limits the use of intraoperative $\mathrm{C}$-arms, so that it cannot perform fluoroscopic positioning during surgery. This type of robot does not integrate any navigation or positioning systems to compensate for these drawbacks. The $2 \mathrm{~B}$-type robots that have been reported thus far do not have reliable safety control strategies. Whether the lamina is about to be cut through or not depends on the experience of the operator. Therefore, we believe that the Nathoo B type robot may be more suitable for the application of minimally 
invasive surgery.

The Nathoo C type robots reported thus far have the same design concept as the surgeon-operated conventional operation. The robot plays the role of an "insurance policy". The members of Surgicobot's research and development team have explained why they did not chose automatic methods to achieve robot-assisted laminectomy: "The surgeon does not see what is happening during the course of the operation because the robot obstructs his view, and there can be errors due to the model not quite corresponding to the reality" (28). Therefore, the team chose a solution that combines human flexibility and robot safety. However, we subscribe to the premise that if the safety strategy is sufficiently effective, it is feasible for the robot to perform the laminectomy automatically. In the future, we believe that the robot architecture in this field should still move in the direction of the Nathoo A type.

Almost all robotic bone cutting tools in the literature were milling drills, and only a single study examined ultrasonic bone cutters (43). In fact, ultrasonic osteotome has many advantages as a bone cutting device. It boasts high accuracy and convenient operation, and enables easy-toperform bone resection operation in complex anatomical locations. The principle involves using high-frequency ultrasonic microvibration to cut mineralized tissue, so the cutting efficiency for bone is high, while the cutting efficiency for soft tissues, such as vascular nerves, is low. Therefore, it has the effect of "cutting hard but not cutting soft" to some extent (44). There are even reports in the literature that ultrasonic bone cutters have a hemostatic function during bone cutting (45). The above characteristics give ultrasonic bone cutters a good performance in the practice of spine surgery laminectomy $(46,47)$. We believe that it has promising application prospects in the field of robot-assisted laminectomy.

For bone cutting strategies, the key point is to choose reasonable cutting parameters including cutting depth, feed rate, and cutting tool settings. At present, a large number of researchers have performed milling layer by layer, which presents a problem that in one layer of the lamina milling process, 2 types of bones-cortical bone and cancellous bone-may appear simultaneously. Moreover, in the instance of approaching the inner wall of the lamina, this milling method may cause some lamina to be penetrated and some not, which is catastrophic for the surgery. The relevant research has mainly focused on path control or force control. In clinical practice, temperature control is also very important. Excessively high temperatures may cause thermal damage to the tissue and even tool deformation, affecting the positioning accuracy and potentially leading to surgery failure.

The safety strategy of robotic-assisted laminectomy revolves around ensuring that the robot does not penetrate the lamina during the cutting process. In order to achieve this, the robot needs to have the ability of state recognition, so it can differentiate between the inner cortical bone and cancellous bone the moment before penetration, and stop or continue accordingly. However, the current state recognition strategy mostly relies on a single parameter, and may thus be susceptible to recognition error. In the future, multi-parameter state recognition should be developed to improve the accuracy of recognition. Also, merely ensuring that the inner cortical layer is not penetrated is not sufficient. The heat produced by the cutting tool during operation can be transmitted through the lamina to the nerve root or spinal cord before the lamina is cut through. If the temperature is too high, the operation becomes unsafe. Therefore, the safety strategy developed in future studies needs to be more comprehensive. Furthermore, few articles have reported on the issues of accuracy, e.g., whether or not the position, angle, and depth of the laminectomy are consistent with planning. In the field of robot-assisted pedicle screw fixation, many clinical applications have been developed. The Gertzbein-Robbins scale (48) was used in most studies to evaluate the accuracy of pedicle screw placement. In addition, some studies also compared the trajectory with preoperative planning (49). However, in the field of laminectomy, there is no unified quantitative standard for the accuracy of laminectomy, and the most accepted standard is non-penetration of the inner cortical bone; however, the definition of the degree of non-penetration has not been specified, which makes it impossible to make a direct comparison between the results of different studies. In this regard, our view is that subsequent research needs to propose an evaluation method, which should include at least 3 dimensions: (I) the general precision of the robotic system as a whole; (II) whether or not the inner cortical bone is intact after robotassisted laminectomy, and classification according to the remaining thickness; (III) a comparison between the actual cutting track and the planned track, such as for the angle of the cut or the volume of the cut lamina. In the future, with the optimization of robot design and algorithms, the accuracy of robots will gradually improve. In combination 
with the development of multi-parameter state recognition, this will increase the safety of these surgeries.

The clinical application scenario for the present studies is robot-assisted spinal open surgery. Robot-assisted minimally invasive spinal surgery and spinal revision surgery have a similar theoretical basis as open surgery. Therefore, when robot-assisted spinal open surgery becomes a reality, the application of these latter methods will ensue. At present, the robot has already been digitized, and, after the breakthrough of open surgery, it is to be expected that minimally invasive robotic surgery will shortly follow. The emphasis in the future will be on how to achieve intelligent robotic surgery. According to the robot automation classification standard proposed by Yang (levels 0-5) (50), the degree of automation in the field of spinal robotics is $0-2$ level at present. The most advanced automation level 2 robot can automatically execute the program set by the operator. Robots need to be gradually developed to nearfull automation, which may include robots conducting surgery planning, medical decision-making, and performing operations completely automatically. Such advanced automation is the embodiment of intelligence. In this way, we will embrace an era of digital, minimally invasive, precise, and intelligent spinal surgery.

As a systematic review, our study was limited by the possibility of an incomplete literature search. Therefore, we expanded the database to be as comprehensive as possible.

\section{Conclusions}

This article reviewed the progress in robot-assisted laminectomy in spinal surgery, and summarized it from 3 perspectives: the robotic systems, bone cutting strategies, and state recognition strategies. The current research has focused more on the latter 2 perspectives. Although some studies have been able to perform laminectomy without penetrating the inner cortex of the lamina, most of these are in vitro experiments. No robots for laminectomy have been made commercially available thus far, and the body of existing research is still some way off from clinical application.

\section{Acknowledgments}

Funding: This work was funded by National Key Research and Development Program of China (no. 2018YFB1307600).

\section{Footnote}

Reporting Checklist: The authors have completed the PRISMA reporting checklist Available at http://dx.doi. org/10.21037/atm-20-5270

Conflicts of Interest: All authors have completed the ICMJE uniform disclosure form (available at http://dx.doi. org/10.21037/atm-20-5270). The authors have no conflicts of interest to declare.

Ethical Statement: The authors are accountable for all aspects of the work in ensuring that questions related to the accuracy or integrity of any part of the work are appropriately investigated and resolved.

Open Access Statement: This is an Open Access article distributed in accordance with the Creative Commons Attribution-NonCommercial-NoDerivs 4.0 International License (CC BY-NC-ND 4.0), which permits the noncommercial replication and distribution of the article with the strict proviso that no changes or edits are made and the original work is properly cited (including links to both the formal publication through the relevant DOI and the license). See: https://creativecommons.org/licenses/by-nc-nd/4.0/.

\section{References}

1. D'Souza M, Gendreau J, Feng A, et al. Robotic-Assisted Spine Surgery: History, Efficacy, Cost, And Future Trends. Robot Surg 2019;6:9-23.

2. Cole AP, Trinh QD, Sood A, et al. The Rise of Robotic Surgery in the New Millennium. J Urol 2017;197:S213-5.

3. Peters BS, Armijo PR, Krause C, et al. Review of emerging surgical robotic technology. Surg Endosc 2018;32:1636-55.

4. Jiang B, Azad TD, Cottrill E, et al. New spinal robotic technologies. Front Med 2019;13:723-9.

5. Nathoo N, Cavusoglu MC, Vogelbaum MA, et al. In touch with robotics: neurosurgery for the future. Neurosurgery 2005;56:421-33; discussion 433.

6. Wang T, Luan S, Hu L, et al. Force-based control of a compact spinal milling robot. Int J Med Robot 2010;6:178-85.

7. Dai Y, Xue Y, Zhang J. Vibration-Based Milling Condition Monitoring in Robot-Assisted Spine Surgery. IEEE/ ASME Transactions on Mechatronics 2015;20:3028-39.

8. Deng Z, Zhang H, Guo B, et al. Hilbert-Huang 
Transform Based State Recognition of Bone Milling with Force Sensing. 2013 IEEE International Conference on Information and Automation. 2013.

9. Wang S, Gao P, Fan L, et al. A bone milling robot for spinal surgery. Journal of Medical Devices, Transactions of the ASME 2016;10.

10. Sun Y, Wang L, Jiang Z, et al. State recognition of decompressive laminectomy with multiple information in robot-assisted surgery. Artif Intell Med 2020;102:101763.

11. Deng Z, Jin H, Hu Y, et al. Fuzzy force control and state detection in vertebral lamina milling. Mechatronics 2016;35:1-10.

12. Ponnusamy K, Chewning S, Mohr C. Robotic approaches to the posterior spine. Spine (Phila Pa 1976) 2009;34:2104-9.

13. Lei L. Research on bilateral control of master-slave robotic spinal surgical system and temperature field in bone grinding [master's thesis]. [Harbin]: Harbin Institute of Technology; 2016. 62p.

14. Hein A, Lueth TC. Control algorithms for interactive shaping surgical robots. Proceedings 2001 ICRA. IEEE International Conference on Robotics and Automation. 2001.

15. Francoise V, Sahbani A, Morel G, et al. A comanipulation device for orthopedic surgery that generates geometrical constraints with real-time registration on moving bones. 2011 IEEE International Conference on Robotics and Biomimetics; 2011 7-11 Dec. 2011.

16. Dai Y, Xue Y, Zhang J. Milling State Identification Based on Vibration Sense of a Robotic Surgical System. IEEE Transactions on Industrial Electronics 2016;63:6184-93.

17. Dai Y, Xue Y, Zhang J, et al., editors. Biologicallyinspired auditory perception during robotic bone milling. 2017 IEEE International Conference on Robotics and Automation (ICRA); 201729 May-3 June 2017.

18. Dai Y, Xue Y, Zhang J. Bioinspired Integration of Auditory and Haptic Perception in Bone Milling Surgery. IEEE/ ASME Transactions on Mechatronics 2018;23:614-23.

19. Jin $\mathrm{H}$, Zhang $\mathrm{P}, \mathrm{Hu} \mathrm{Y}$, et al., editors. Design and kinematic analysis of A Pedicle Screws Surgical Robot. 2010 IEEE International Conference on Robotics and Biomimetics; 2010 14-18 Dec. 2010.

20. Jin $\mathrm{H}$, Wang $\mathrm{L}, \mathrm{Hu} \mathrm{Y}$, et al., editors. Design and control strategy of robotic spinal surgical system. The 2011 IEEE/ ICME International Conference on Complex Medical Engineering; 2011 22-25 May 2011.

21. Jin H, Hu Y, Li F, et al., editors. Safety Design and Control Algorithm for Robotic Spinal Surgical System. 2011 First International Conference on Robot, Vision and
Signal Processing; 2011 21-23 Nov. 2011.

22. Wang R. Design and Research on the Surgical Robot of Laminate Grinding [master's thesis]. [Hebei]: Hebei University of Engineering; 2017. 71p.

23. Wang R, Wang N, Wang L. Design of robot for lamina grinding operation. KeXue JiShu Chuangxin/Scientific and technological innovation 2017;(34):188-9. Chinese.

24. Sun Y, Jiang Z, Qi X, et al. Robot-Assisted Decompressive Laminectomy Planning Based on 3D Medical Image. IEEE Access 2018;6:22557-69.

25. Fan L. Design and analysis of a compact robotic surgical system for vertebral lamina grinding task based on force feedback [master's thesis]. [Harbin]: Harbin Institute of Technology; 2016. 57p.

26. Childers CP, Maggard-Gibbons M. Estimation of the Acquisition and Operating Costs for Robotic Surgery. JAMA 2018;320:835-6.

27. Trybula SJ, Oyon DE, Wolinsky JP, et al. Robotic Tissue Manipulation and Resection in Spine Surgery. Neurosurg Clin N Am 2020;31:121-9.

28. Kochan A. A cobotic solution for surgical applications. Industrial Robot-an International Journal 2004;31:478-80.

29. Bonneau E, Taha F, Gravez P, et al. Surgicobot: Surgical gesture assistance cobot for maxillo-facial interventions. Perspective in Image-Guided Surgery 2004:353-360.

30. Van Rhijn J, Riwan A, Jabbour Z, et al. Mechanical Impedance: A Cobotic and Haptic Actuators Performance Criterion. In: Kappers AML, VanErp JBF, Tiest WMB et al., editors. Haptics: Generating and Perceiving Tangible Sensations, Pt I, Proceedings. Lecture Notes in Computer Science, 2010. p. 225-232.

31. Riwan A, Giudicelli B, Taha F, et al. Surgicobot project: Robotic assistant for spine surgery. Irbm 2011;32:130-4.

32. Zhang J, Wang T, Luan S, et al. A fuzzy logic based control method for spine robot. Cailiao Kexue yu Gongyi/ Material Science and Technology 2006;14:77-82. Chinese.

33. Qi X, Sun Y, Ma X, et al. Multilevel Fuzzy Control Based on Force Information in Robot-Assisted Decompressive Laminectomy. Adv Exp Med Biol 2018;1093:263-79.

34. Ma X. Research on path plan and safety control for robotassisted vertebral lamina milling [master's thesis]. [Harbin]: Harbin Institute of Technology; 2017. 60p.

35. Fan L, Gao P, Zhao B, et al. Safety control strategy for vertebral lamina milling task. CAAI Transactions on Intelligence Technology 2016;1:249-58.

36. Jiang Z, Qi X, Sun Y, et al. Cutting Depth Monitoring Based on Milling Force for Robot-Assisted Laminectomy. IEEE Transactions on Automation Science and 
Engineering 2020;17:2-14.

37. Dai Y, Xue Y, Zhang J. Bone milling condition monitoring based on sound signal processing. Journal of Vibration and Shock 2015;34:19-23.

38. Zhu J. State perception and safety control of grinding process in spine surgery robot [master's thesis]. [Harbin]: Harbin Institute of Technology; 2017. 57p.

39. Du W, Sun T, Ding Y, et al. Robot-assisted treatment of unstable pelvic fractures with a percutaneous iliac lumbar double rod fixation combined with a percutaneous pelvic anterior ring INFIX fixation. Int Orthop 2020;44:1223-32.

40. Goo Bong C, Soo Gang L, Sungmin K, et al., editors. A robot-assisted surgery system for spinal fusion. 2005 IEEE/RSJ International Conference on Intelligent Robots and Systems; 2005 2-6 Aug. 2005.

41. Lee J, Hwang I, Kim K, et al. Cooperative robotic assistant with drill-by-wire end-effector for spinal fusion surgery. Industrial Robot 2009;36:60-72.

42. Sun Y, Jiang Z, Qi X, et al. Model-based spinal deformation compensation in robot-assisted decompressive laminectomy. Mechatronics 2019;59:115-26.

43. Li K, Sun Y, Li M, et al., editors. Control Method of Constant Force Grinding based on Active and Passive Control*. 2019 IEEE International Conference on

Cite this article as: $\mathrm{Li}$ Z, Yu G, Jiang S, Hu L, Li W. Robotassisted laminectomy in spinal surgery: a systematic review. Ann Transl Med 2021;9(8):715. doi: 10.21037/atm-20-5270
Robotics and Biomimetics (ROBIO); 2019 6-8 Dec. 2019.

44. Labanca M, Azzola F, Vinci R, et al. Piezoelectric surgery: twenty years of use. Br J Oral Maxillofac Surg 2008;46:265-9.

45. Sanborn MR, Balzer J, Gerszten PC, et al. Safety and efficacy of a novel ultrasonic osteotome device in an ovine model. J Clin Neurosci 2011;18:1528-33.

46. Duerr FM, Seim HB 3rd, Bascunan AL, et al. Piezoelectric surgery -a novel technique for laminectomy. J Invest Surg 2015;28:103-8.

47. Al-Mahfoudh R, Qattan E, Ellenbogen JR, et al. Applications of the ultrasonic bone cutter in spinal surgery--our preliminary experience. Br J Neurosurg 2014;28:56-60.

48. Gertzbein SD, Robbins SE. Accuracy of pedicular screw placement in vivo. Spine (Phila Pa 1976) 1990;15:11-4.

49. Jiang B, Karim Ahmed A, Zygourakis CC, et al. Pedicle screw accuracy assessment in ExcelsiusGPS® robotic spine surgery: evaluation of deviation from pre-planned trajectory. Chin Neurosurg J 2018;4:23.

50. Yang GZ, Cambias J, Cleary K, et al. Medical roboticsRegulatory, ethical, and legal considerations for increasing levels of autonomy. Sci Robot 2017;2:2. 
Table S1 Search strategy in the 8 databases to identify studies associated to robotic-assisted laminectomy

\section{PubMed}

Search strategy

\#1 'Decompression, Surgical' [MeSH Terms] OR 'Surgical Decompression' [Title/Abstract] OR 'Decompressions, Surgical' [Title/Abstract] OR 'Surgical Decompressions' [Title/Abstract]

\#2 Laminectomy [MeSH Terms] OR Laminectomies [Title/Abstract] OR Laminotomy [Title/Abstract] OR Laminotomies [Title/Abstract]

\#3 \#1 OR \#2

\#4 'Robotic Surgical Procedures' [MeSH Terms] OR Robot [Title/Abstract] OR robotic [Title/Abstract]

\#5 \#3 AND \#4

Access Date: from 01/02/2020 to 01/03/2020

Published time: from 01/01/2000 to 01/03/2020

EMBASE

\section{Search strategy}

\#1 'robotic spine surgical system'/exp

\#2 'robotics'/exp

\#3 'robot' OR 'robotic' OR 'robot',

\#4 \#1 OR \#2 OR \#3

\#5 'laminectomy'/exp

\#6 'spinal cord decompression'/exp

\#7 'never decompression'/exp

\#8 'laminectomy' OR 'decompression'

$\# 9$ \#5 OR \#6 OR \#7 OR \#8

\#10 \#4 AND \#9

Access Date: from 01/02/2020 to 01/03/2020

Published time: from $01 / 01 / 2000$ to $01 / 03 / 2020$

The Cochrane Library

Search strategy

\#1 (Laminectom*):ti,ab,kw

\#2 (Laminotom*):ti,ab,kw

\#3 (Decompression):ti,ab,kw

\#4 \#1 or \#2 or \#3

\#5 (robot*):ti,ab,kw

\section{\#6 \#4 AND \#5}

Access Date: from 01/02/2020 to 01/03/2020

Published time: from 01/01/2000 to 01/03/2020

Web of Science

Search strategy

\#1 TOPIC: Laminectom* or Laminotom* or Decompression

\#2 TOPIC: Robot*

\#3 \#1 AND \#2

$\# 4$ \#3 with the exception of patent

Access Date: from 01/02/2020 to 01/03/2020

Published time: from 1900 to 2020

\section{EEE Xplore}

Search strategy

\#1 (Full Text \& Metadata:Laminectomy or Laminectomies or Laminotomy or Laminotomies)

\#2 ("Full Text \& Metadata":"Surgical Decompression")

\#3 ("Mesh_Terms":Decompression, Surgical)

\#4 \#1 OR \#2 OR \#3

\#5 ("Full Text \& Metadata"::robot*)

\section{\#6 \#4 AND \#5}

Access Date: from 01/02/2020 to 01/03/2020

Published time: from 1872 to 2020

CNKI

Search strategy:

(SU=' 椎板切除术 '+' 椎板减压术 '+' 椎板成形术 ') AND (SU=' 机器人手术 '+' 机器人')

Access Date: from 01/02/2020 to 01/03/2020

Published time: from 2000 to 2020

Wanfang

Search strategy

(主题: ( 椎板切除术) + 主题: (椎板减压术) + 主题:(椎板成形术)) AND (主题:(机器人手术)+主题:(机器人)) Access Date: from 01/02/2020 to 01/03/2020

Published time: from 2000 to 2020

SinoMed

Search strategy

(椎板切除术 $O R$ 椎板减压术 $O R$ 椎板成形术) AND (机器人手术 $O R$ 机器人)

Access Date: from 01/02/2020 to 01/03/2020

Published time: from 2000 to 2020 